\title{
COLOR Y ESTADO DE MADUREZ DEL FRUTO DE TOMATE DE CÁSCARA ${ }^{1}$
}

\author{
Pedro Benito-Bautista ${ }^{2}$, Nelly Arellanes-Juárez ${ }^{2}$, María Eufemia Pérez-Flores ${ }^{2}$
}

\section{RESUMEN}

Color y estado de madurez del fruto de tomate de cáscara. Este estudio tuvo como objetivo identificar las características físico-químicas que determinan el estado de madurez del fruto de cuatro variedades de tomate de cáscara. Se recolectaron 100 frutos de las variedades: San Martín, Tecozautla 04, Rendidora y Diamante, sembrados a una densidad de 40000 plantas/ha, en ambiente protegido en el campo experimental del Centro Interdisciplinario de Investigación para el Desarrollo Integral Regional (CIIDIR)Instituto Politécnico Nacional (IPN) - Unidad Oaxaca, México, en los ciclos verano-otoño 2013 y primaveraverano 2014, considerando: el llenado del cáliz y el tiempo transcurrido desde el amarre del mismo. Los frutos fueron almacenados a temperatura ambiente $\left(25 \pm 3{ }^{\circ} \mathrm{C}\right)$, retirando cada semana, diez frutos de cada variedad para determinar: pérdida de peso, resistencia a la penetración, sólidos solubles, color, $\mathrm{pH}$ y acidez titulable. Los parámetros de color: a*, h* e índice de color (IC), fueron estadísticamente significativos, y presentaron alta correlación con las propiedades físicas y químicas en función de la maduración. La coordenada a* presentó el mayor coeficiente de correlación con la resistencia a la penetración del fruto, seguida por el IC y después el valor $\mathrm{h}^{*}$. A su vez, a* e IC mostraron alta relación con las variables: pérdida de peso, acidez titulable, sólidos solubles y $\mathrm{pH}$. De acuerdo a los resultados, el parámetro de color con mayor correlación con las características de calidad fue $\mathrm{a}^{*}$, y las variedades con mejores características de calidad fueron: Tecozautla 04 y San Martín.

Palabras claves: índice de color en frutos, calidad de frutos, Physalis ixocarpa.

\begin{abstract}
Color and state of maturity of fruit of the husk tomato. The present study aimed to identify the physical and chemical characteristics that determine the state of maturity of the fruit of four varieties of husk tomato. One hundred fruits of the varieties: San Martin, Tecozautla 04, Rendidora, and Diamante, were collected. They were cultivated at a density of 40,000 plants/ha under experimental greenhouse conditions at the Interdisciplinary Research Center for Integrated Regional Development (CIIDIR), National Polytechnic Institute (IPN), in Oaxaca, Mexico. The plants were grown during the summer-autumn 2013 and springsummer 2014 growing cycles. The husk filling and elapsed time since the fruit set were considered as indicators of harvest. Fruits were stored at room temperature $\left(25 \pm 3{ }^{\circ} \mathrm{C}\right)$, and each week 10 fruits of each variety were selected and analyzed for weight loss, penetration resistance, soluble solids, color, $\mathrm{pH}$ and titratable acidity. The statistic results indicate that color parameters $\mathrm{a}^{*}, \mathrm{~h} *$ and color index (IC), were significantly high, and showed a high correlation with the physical and chemical properties for the state of maturation. The $\mathrm{a}^{*}$ color coordinate presented the highest coefficient of correlation with fruit texture, followed by IC and $\mathrm{h}^{*}$. At the same time, a $*$ and CI showed strong correlation with variables weight loss, titratable acidity, $\mathrm{pH}$, and soluble solids. According to these results, the a* color parameter showed the greatest correlation with quality features, and varieties Tecozautla 04 and San Martin had the best quality characteristics.
\end{abstract}

Keywords: color index of fruits, fruit quality, Physalis ixocarpa.

\footnotetext{
1 Recibido: 11 marzo, 2014. Aceptado: 4 de junio, 2015. Este trabajo fue parte del proyecto de investigación SIP20140470: "Estudio de la calidad y cambios químicos y fisiológicos del fruto de tomatillo (Physalis ixocarpa Brot.) en poscosecha", apoyado por el Instituto Politécnico Nacional. Oaxaca, México.

2 Centro Interdisciplinario de Investigación para el Desarrollo Integral Regional- Instituto Politécnico Nacional-Unidad Oaxaca, México. C.P. 71230.pbenito@ipn.mx,narellanes@ipn.mx,mperezf@ipn.mx
} 


\section{INTRODUCCIÓN}

El color es una percepción humana de la luz reflejada por un objeto, una apreciación que depende de cómo nuestros ojos detectan esta luz y de cómo nuestro cerebro la procesa. Por esta razón, para cuantificar y estandarizar el color, se han desarrollado equipos y modelos cromáticos que describen matemáticamente la representación de los colores utilizando espectrofotómetros o colorímetros (Yam y Papadakis, 2004).

El espacio de color CIE-L*a*b* describe todos los colores que puede percibir el ojo humano a partir de sus tres coordenadas: $\mathrm{L}^{*} \mathrm{a}^{*} \mathrm{~b}^{*}$, y es aceptado internacionalmente para evaluar el color en los alimentos y otros productos industrializados (León et al., 2006; Pérez-López et al., 2009; Jha et al., 2010; Pathare et al., 2013). Otro de los sistemas utilizado para trasmitir los valores de color, es el espacio CIE L*C* $\mathrm{h}$, el cual es una representación polar del sistema CIE L*a*b*, con coordenadas cilíndricas ( $\mathrm{L}^{*} \mathrm{C}^{*} \mathrm{~h} *$ ) en lugar de coordenadas rectangulares (McGuirre, 1992; CarvajalHerrera et al., 2011; Pathare et al., 2013).

En frutos y vegetales, el cambio de color es causado por la degradación de la clorofila y el descubrimiento o síntesis de pigmentos carotenoides en los cloroplastos y cromoplastos, y los pigmentos fenólicos: antocianinas, flavonoles y pro antocianidinas (Lancaster et al., 1997). Este cambio se vincula con la evolución de la textura, aroma y sabor que esperan los consumidores de estos productos en el momento de consumo y se relaciona fuertemente con la resistencia a la penetración, atributo de calidad que denota frescura del producto (Surmacka, 2002), por lo que es utilizado como un indicador de madurez de los frutos (Barrett et al., 1998).

Durante la maduración de los frutos ocurren cambios fisiológicos, bioquímicos y estructurales que influyen en la producción de compuestos químicos que generan el sabor y aroma correspondientes. El ablandamiento de los tejidos varía de acuerdo a la especie y variedad del fruto, y se debe a la degradación de pectinas y hemicelulosas de la pared celular por acción de diferentes enzimas hidrolíticas (Karakurt, 2007). Las características sensoriales, principalmente de olor y sabor, juegan un papel central en la calidad de los frutos y dependen del gusto y aroma percibidos por cada consumidor. En frutos y hortalizas son modificados por la variedad y momento de la maduración de cada especie (Kader,
2009), y están ligadas a la biosíntesis y a una variación de concentraciones o modificaciones de varios compuestos químicos, principalmente azúcares, ácidos, taninos y aceites esenciales (Dávila-Aviña et al., 2011; Torres et al., 2013).

Por esta razón, varios investigadores han propuesto la utilización de algunos parámetros de color que permitan una correlación directa entre el aspecto visual de frutas y vegetales y sus cambios durante la maduración (Fischer y Martínez, 1999; Ayala-Silva et al., 2005; Carvajal-Herrera et al., 2011; Tapre y Jain, 2012). En tomate (Solanum lycopersicon Mill.), se han reportado variaciones significativas en la coordenada $a^{*}$, vinculada con la degradación de clorofila y la síntesis de licopeno que permiten diferenciar seis etapas de maduración (López-Camelo y Gómez, 2004). En mango (Mangifera indica L.), las diferentes expresiones del cambio de color son una herramienta útil para diferenciar variedades y etapas de maduración (Ayala-Silva et al., 2005; Jha et al., 2006; Jha et al., 2010; Quintero et al., 2013). En banano (Musa spp.), el cambio de color de verde a amarillo causa incremento en los valores de $a^{*}$ y $\mathrm{b}^{*}$, y disminuciones en el ángulo de matiz $\left(\mathrm{h}^{*}\right)$, lo que faculta el establecimiento de cartas comerciales estándar de color para diferenciar siete etapas de maduración, traducidas a una escala numérica (OsunaGarcía et al., 2007; Salvador et al., 2007; Amnuaysin et al., 2012; Tapre y Jain, 2012). En guayaba (Psidium guajava L.), el cambio de color del epicarpio por medio de la disminución del valor de matiz $\left(\mathrm{H}^{\circ}\right)$ e incremento del valor de croma $\left(\mathrm{C}^{*}\right)$, establecen el grado de maduración de este fruto (González-Cárdenas et al., 2010). En papaya, el cambio en el color del epicarpio permite identificar seis etapas de madurez, en las cuales, los valores de la coordenada $b *$ delimita las etapas de madurez temprana, mientras que los de $a^{*}$ señalan las etapas finales afectando el valor de tono $\mathrm{H}^{\circ}$ (Santamaría-Basulto et al., 2009a; 2009b).

En tomate de cáscara (Physalis ixocarpa Brot.), especie nativa de México y Centroamérica (SantiaguilloHernández y Blas-Yáñez, 2009), cultivada desde la época prehispánica, no se ha definido un indicador apropiado para la maduración (Cruz-Álvarez et al., 2012), y la determinación del color del fruto ha sido relativamente poco investigada de manera objetiva y con métodos cuantitativos en comparación con otros productos hortofrutícolas. Esto pese a que este fruto goza de fuerte demanda en el mercado, tanto por sus 
propiedades sensoriales y alimenticias, como por sus cualidades funcionales y terapéuticas provenientes de su contenido de carotenoides, fenoles y ácido ascórbico, entre otros (Bao-Ning et al., 2002; Choi et al., 2006; Ibave-González y Ochoa, 2007; González-Mendoza et al., 2011). Para su caracterización y comercialización, en México se sigue la Norma Mexicana NMX-FF-54-1982. - Productos alimenticios no industrializados para uso humano -hortalizas en estado fresco- como tomate con cáscara (SECOFI, 1982), que se basa principalmente en el tamaño y la apariencia del fruto, dejando a un lado el valor nutricional, el grado de maduración y otras características químicas y bioquímicas que apoyen beneficios para la salud humana y su comportamiento poscosecha y agroindustrial.

Los valores de peso, tamaño, firmeza, acidez y sólidos solubles, pueden ser utilizados como criterios para definir la calidad del fruto (Torres-Panduro, 1998; Santiaguillo-Hernández et al., 2004; JiménezSantana et al., 2012). Los escasos trabajos en relación al color, reportaron que después de la cuarta semana de crecimiento del fruto, los niveles de clorofila y carotenos decrecen (Cantwell et al., 1992), esta información puede asociarse con el color del epicarpio (Azcón-Bieto y Talón, 2000), lo cual puede ser una alternativa para establecer un indicador de madurez no destructivo para este fruto. En uchuva (Physalis peruviana L.), fruto de otra especie de este género, se han establecido seis etapas de madurez utilizando el color del epicarpio (Fischer y Martínez, 1999).

Considerando que el color es una característica externa y un método no destructivo para determinar el grado de maduración de varios frutos, el objetivo de este estudio fue determinar el parámetro de color del epicarpio que guarda la mayor relación con características fisicoquímicas que determinan el estado de madurez del fruto de cuatro variedades de tomate de cáscara.

\section{MATERIALES Y MÉTODOS}

Se obtuvieron cien frutos de cuatro variedades de tomate de cáscara: San Martín, Tecozautla 04, Rendidora y Diamante, las cuales fueron sembrados a una densidad de 40000 plantas/ha en ambiente protegido, en el área del campo experimental del Centro Interdisciplinario de Investigación para el Desarrollo Integral Regional (CIIDIR), del Instituto Politécnico Nacional (IPN), Unidad Oaxaca, en los ciclos verano-otoño 2013 y primavera verano 2014, con temperaturas promedio de $18-22^{\circ} \mathrm{C}$ y humedad relativa de $60-75 \%$. Los frutos fueron cosechados considerando como indicadores de madurez el llenado del cáliz y el tiempo transcurrido desde el amarre del mismo. Estos fueron llevados al laboratorio, donde se les eliminó el cáliz, se lavaron con agua y desinfectaron con una solución de cloro de 25 ppm; posteriormente, se dejaron secar por doce horas a temperatura ambiente. Luego se formaron grupos de diez frutos y se colocaron en charolas, almacenándose a temperatura ambiente $\left(25 \pm 3{ }^{\circ} \mathrm{C}\right)$. Cada semana se retiró un lote de cada variedad para el análisis de las variables pérdida de peso, resistencia a la penetración, sólidos solubles, color, $\mathrm{pH}$ y acidez titulable.

La metodología de análisis fue la descrita por la AOAC (2010) para pérdida de peso, sólidos solubles y acidez titulable.

La determinación del peso de los frutos se realizó con una balanza analítica. La pérdida de peso se calculó por la diferencia entre el primer y segundo valor multiplicado por 100 y dividido entre el peso inicial.

El contenido de sólidos solubles se midió con un refractómetro modelo Automatic y se reportó como ${ }^{\circ}$ Brix.

La acidez titulable se obtuvo extrayendo $5,0 \mathrm{~g}$ de muestra homogénea de tejido, con $50 \mathrm{ml}$ de agua destilada. El conjunto se valoró con hidróxido de sodio $0,1 \mathrm{~N}$, utilizando fenolftaleína al 0,1\% como indicador. Los resultados se expresaron como porcentaje de ácido cítrico.

Para la determinación del $\mathrm{pH}$ se utilizó un potenciómetro.

La pérdida de resistencia a la penetración se realizó mediante un medidor de textura, modelo TATX2i con una sonda de $0,5 \mathrm{~cm}$ de diámetro en dos puntos extremos del eje ecuatorial de cada fruto y los resultados se expresaron en Newtons $(\mathrm{N})$.

Los parámetros de color L*, a* y b* (CIELAB), fueron evaluados con un medidor de color modelo Mini Scan, previamente calibrado y los resultados se transformaron a valores de matiz $\left(\mathrm{h}^{*}\right)$ mediante el cálculo del arco tangente de $\left(\mathrm{b}^{*} / \mathrm{a}^{*}\right)$; croma (C) utilizando el cálculo de $\left[\left(\mathrm{a}^{* 2}+\mathrm{b}^{* 2}\right)^{1 / 2}\right]$, de acuerdo con McGuirre (1992), y el índice de color (IC): [(a*x 1000)/ (Lx b*)], de acuerdo con Francis y Clydesdale (1975).

A los resultados obtenidos para cada variable se les realizó un análisis de varianza y las medias se compararon con la prueba de Tukey $(\mathrm{p} \leq 0,05)$ usando el programa Statgraphics Centurion XVI.II (Statistical Graphics Corp.). 


\section{RESULTADOS Y DISCUSIÓN}

\section{Color}

La percepción visual fue diferente entre las variedades evaluadas y el periodo de evaluación (Figura 1).

\section{Coordenada a*}

El análisis estadístico de este parámetro, bajo las condiciones del estudio, mostró diferencias significativas $(\mathrm{p} \leq 0,05)$ entre variedades, indicando que las variedades San Martín y Tecozautla 04 tendieron a desarrollar y mantener mayor color verde $\left(a^{*}=9,94\right)$, mientras que las variedades Rendidora y Diamante $\left(\mathrm{a}^{*}=9,15\right)$, tendieron a perder el color verde (clorofila) (Figuras 1 y 2). Estos resultados pudieron deberse a los diferentes tiempos de degradación de la clorofila en cada variedad, por su contenido y actividad de clorofilasas y la presencia de otras enzimas degradantes, o a la facilidad de degeneración de las estructuras cloroplástidas (Hornero-Méndez y Mínguez-Mosquera, 2002).

El periodo de almacenamiento modificó los valores de la coordenada $\mathrm{a}^{*}$, el análisis estadístico mostró diferencias significativas $(\mathrm{p} \leq 0,05)$ entre las diferentes fechas de muestreo (Figura 3). Se observó que los valores de $a^{*}$ se redujeron a medida que avanzó el periodo de almacenamiento pasando de un valor de $-14,1$, para el momento de corte, hasta alcanzar el valor de $-7,0$ en la quinta semana de almacenamiento. Este comportamiento fue similar al reportado para el fruto de Physalis peruviana L., en el cual el cambio de color permitió determinar seis etapas de maduración, que representan matices de color verde en el momento de corte, a tonos de naranja-rojo en su estado de madurez de consumo (Fischer y Martínez, 1999). De igual manera se han indicado tendencias con correlaciones positivas, entre el parámetro $\mathrm{a}^{*} \mathrm{y}$ las diversas etapas de maduración en banana (Soltani et al., 2011) y mango "Keitt" (Padda et al., 2011). Lo anterior se explica por el catabolismo de la clorofila, debido a la acción de clorofilasas y la aparición de

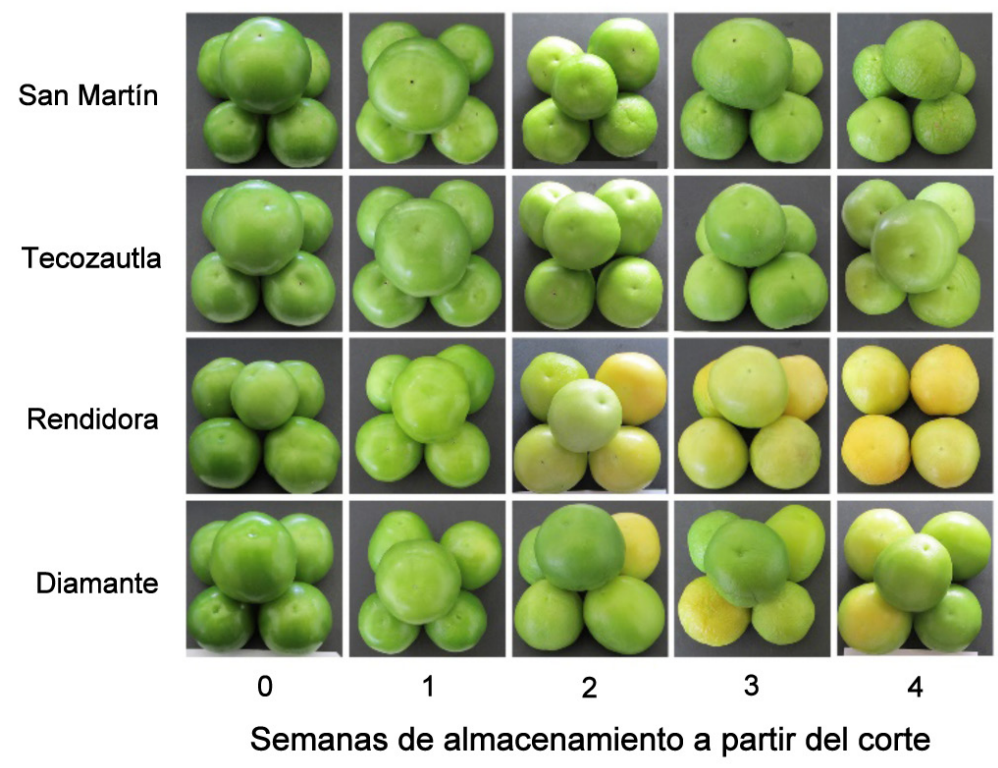

Figura 1. Cambio de color en variedades de Physalis ixocarpa Brot., durante su maduración a temperatura ambiente $\left(25 \pm 3^{\circ} \mathrm{C}\right)$, a partir de la cosecha del fruto, durante los ciclos verano-otoño 2013 y primavera-verano 2014. Oaxaca, México.

Figure 1. Change in Physalis ixocarpa Brot. varieties, during its ripening at room temperature $\left(25 \pm 3^{\circ} \mathrm{C}\right)$, after the fruit harvest, in summer-autumn 2013 and spring-summer 2014 cycles. Oaxaca, México. 


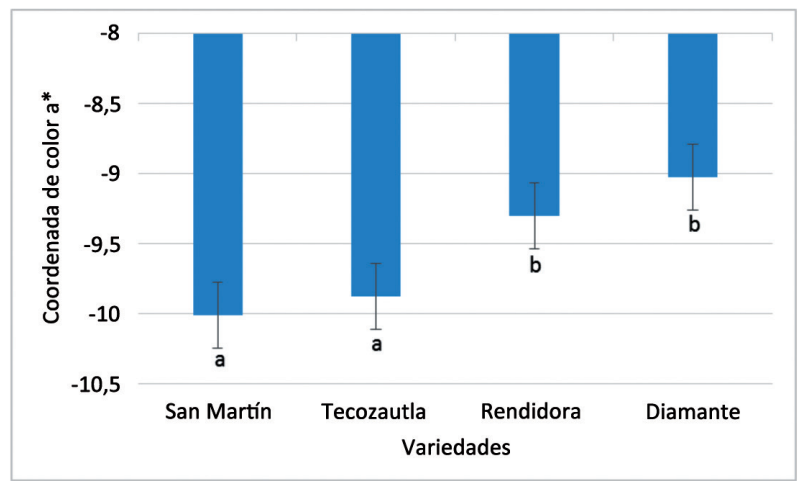

Figura 2. Valor de la coordenada de color a*, de variedades de tomate de cáscara almacenados a temperatura ambiente $\left(25 \pm 3{ }^{\circ} \mathrm{C}\right)$, después de la cosecha del fruto, durante los ciclos verano-otoño 2013 y primavera-verano 2014. Oaxaca, México.

Letras distintas señalan diferencias significativas entre los valores según el método de Tukey $(\mathrm{p}<0,05)$.

Figure 2. Color a* coordinate value of varieties of husk tomato stored at room temperature $\left(25 \pm 3^{\circ} \mathrm{C}\right)$, after the fruit harvest, in summer-autumn 2013 and spring-summer 2014 cycles. Oaxaca, Mexico. Values with different letters are significantly different $(\mathrm{p}<0,05)$, by Tukey Method.

otros pigmentos como carotenoides (Hornero-Méndez y Mínguez-Mosquera, 2002).

\section{Croma $\left(C^{*}\right)$}

El análisis estadístico de los valores de la coordenada cilíndrica $\mathrm{C}^{*}$, mostró diferencias significativas entre las medias correspondientes a las diferentes variedades (Figura 4). La variedad Tecozautla 04 presentó el valor más alto con 35,15 , seguida por la variedad San Martín con 34,28 y finalmente, las variedades Rendidora y Diamante con un promedio de 34,07. Estos datos tienen una relación inversa con los valores de la coordenada $\mathrm{a}^{*}$, ya que $\mathrm{C}^{*}$ se calcula con los cuadrados de los valores $a^{*}$ y $b^{*}$, lo que convierte a los valores negativos en positivos, los valores más bajos de $\mathrm{C}^{*}$ son aquellos cuyos valores de $\mathrm{a}^{*}$ se encuentran cercanos a 0 (Rendidora y Diamante). Así mismo, dado que este parámetro indica la pureza o saturación del color, fueron las variedades San Martín y Tecozautla 04 las que presentaron valores de $\mathrm{C}^{*}$

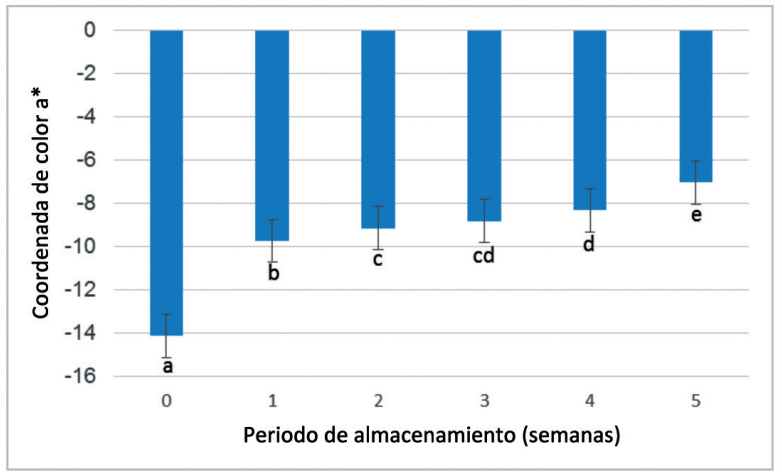

Figura 3. Efecto del tiempo de almacenamiento a $25 \pm 3{ }^{\circ} \mathrm{C}$ sobre la coordenada de color a* en variedades de tomate de cáscara, después de la cosecha del fruto, durante los ciclos verano-otoño 2013 y primaveraverano 2014. Oaxaca, México.

Letras distintas señalan diferencias significativas entre los valores según el método de Tukey $(\mathrm{p}<0,05)$.

Figure 3. Storage time at $25 \pm 3{ }^{\circ} \mathrm{C}$ effect on the $\mathrm{a}^{*}$ color coordinate in husk tomato varieties, after the harvest fruit, in summer-autumn 2013 and springsummer 2014 cycles. Oaxaca, Mexico.

Values with different letters are significantly different $(\mathrm{p}<0,05)$, by Tukey method.

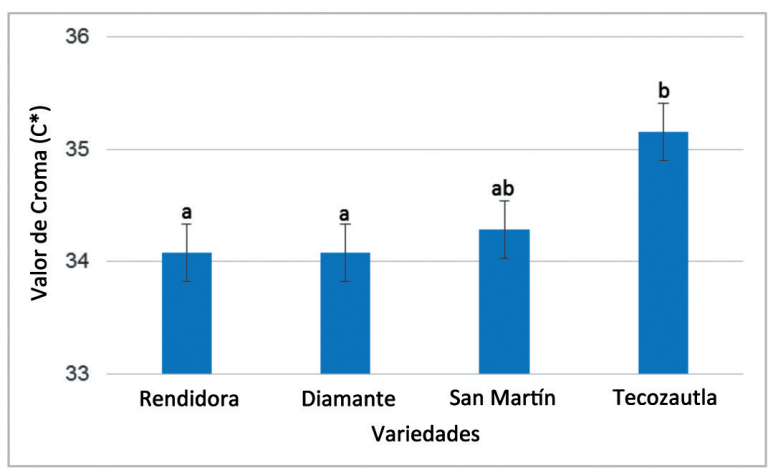

Figura 4. Valor de Croma $\left(\mathrm{C}^{*}\right)$ en variedades de tomate de cáscara almacenado a temperatura ambiente $(25$ $\pm 3{ }^{\circ} \mathrm{C}$ ), después de la cosecha del fruto. Oaxaca, México, durante los ciclos verano-otoño 2013 y primavera-verano 2014. Oaxaca, México.

Letras distintas señalan diferencias significativas entre los valores según el método de Tukey $(\mathrm{p}<0,05)$.

Figure 4. Chroma value $(\mathrm{C} *)$ in husk tomato varieties stored at room temperature $\left(25 \pm 3{ }^{\circ} \mathrm{C}\right)$, after the harvest fruit, in summer-autumn 2013 and springsummer 2014 cycles. Oaxaca, Mexico.

Values with different letters are significantly different $(\mathrm{p}<0,05)$, by Tukey method. 
más altos, por manifestar menor pérdida de clorofila (López-Camelo y Gómez, 2004).

El comportamiento de la coordenada $\mathrm{C}^{*}$ fue afectado por el periodo de almacenamiento de forma moderada (Figura 5), el análisis estadístico mostró un valor de $\mathrm{C}^{*}$ de 34,5 para el momento de corte, el cual se redujo durante las semanas 1,2 y 5 a valores de 32,4, y aumentó en las semanas 3 y 4 a valores de 35,9 y 38,6 , respectivamente. Esta variación se pudo deber a diferentes factores, entre los que se pueden destacar diferencias de color en el momento de corte y la pérdida de clorofila en la semana cinco. Tendencias erráticas similares fueron reportadas para el valor de $C^{*}$ durante la maduración de frutos de banana por Soltani et al. (2011). Para frutos de jitomate, la coordenada $\mathrm{C}^{*}$ no es un indicador adecuado de madurez, ya que esencialmente es una expresión de pureza para un color específico y que, en el caso de este fruto, se presentan simultáneamente tanto degradación de clorofila como síntesis de compuestos carotenoides, lo cual aplicaría también para el tomate de cáscara (Camelo et al., 2004).

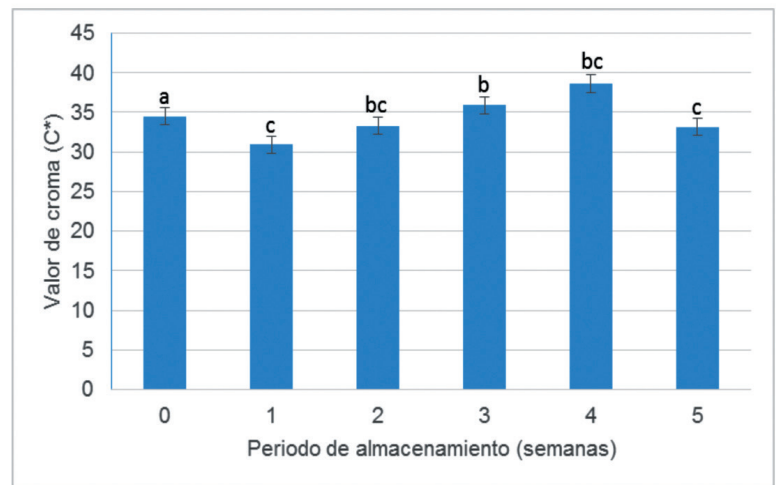

Figura 5. Efecto del periodo de almacenamiento a temperatura ambiente $\left(25 \pm 3{ }^{\circ} \mathrm{C}\right)$, sobre el valor de croma $\left(\mathrm{C}^{*}\right)$ en variedades de tomate de cáscara, durante los ciclos verano-otoño 2013 y primaveraverano 2014. Oaxaca, México.

Letras distintas señalan diferencias significativas entre los valores según el método de Tukey $(\mathrm{p}<0,05)$.

Figure 5. Storage period at room temperature $\left(25 \pm 3{ }^{\circ} \mathrm{C}\right)$ effect on chroma $\left(\mathrm{C}^{*}\right)$ value in husk tomato varieties, in summer-autumn 2013 and springsummer 2014 cycles. Oaxaca, México Values with different letters are significantly different $(\mathrm{p}<0,05)$, by Tukey method.

\section{Ángulo de matiz (h*)}

El análisis estadístico de los valores de color expresado como ángulo de matiz ( $\left.\mathrm{h}^{*}\right)$, mostró diferencias significativas entre variedades, los datos de esta coordenada cilíndrica mostraron un valor alto para la variedad San Martín $(107,7)$, seguida por Tecozautla $04(106,9)$ y al final por las variedades Diamante y Rendidora con un promedio de 105,4; estos valores siguieron una tendencia similar al valor de $C^{*}$ (Figura 6), lo cual indica que los colores de estos frutos cambian gradualmente de verde a amarillo, dependiendo de la variedad (Figura 1).

La expresión de color presentó un comportamiento inversamente proporcional al período de almacenamiento de los frutos (Figura 7), el análisis estadístico mostró el valor más alto $(114,5)$ para las muestras obtenidas en el momento de corte, seguida por las representantes de la semana 1 y 2 con un promedio de 107,7, después las pertenecientes a las semanas 3 y 4 con un valor de 103,7 y finalmente en

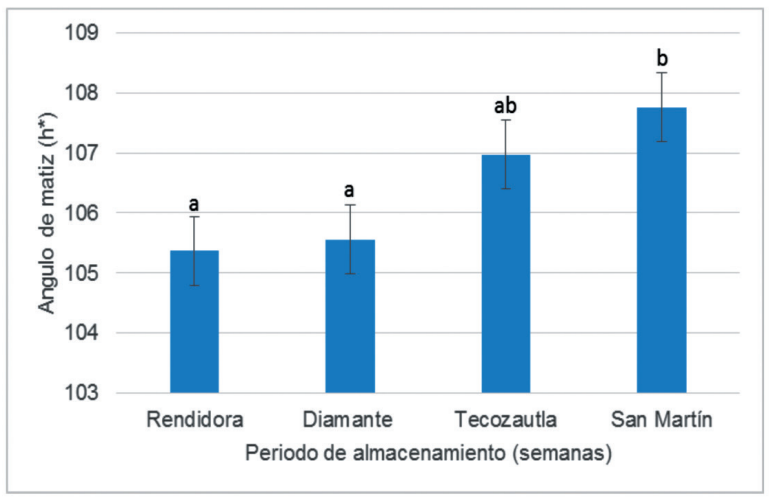

Figura 6. Color expresado como ángulo de matiz ( $\mathrm{h}^{*}$ ) en variedades de tomate de cáscara almacenado a temperatura ambiente $\left(25 \pm 3{ }^{\circ} \mathrm{C}\right)$, después de la cosecha del fruto, durante los ciclos verano-otoño 2013 y primavera-verano 2014. Oaxaca, México. Letras distintas señalan diferencias significativas entre los valores según el método de Tukey $(\mathrm{p}<0,05)$.

Figure 6. Color expressed as hue angle $(\mathrm{h} *)$ in varieties of husk tomato stored at room temperature $(25 \pm$ $3{ }^{\circ} \mathrm{C}$ ), after the harvest fruit, in summer-autumn 2013 and spring-summer 2014 cycles. Oaxaca, México

Values with different letters are significantly different $(p<0,05)$, by Tukey method. 


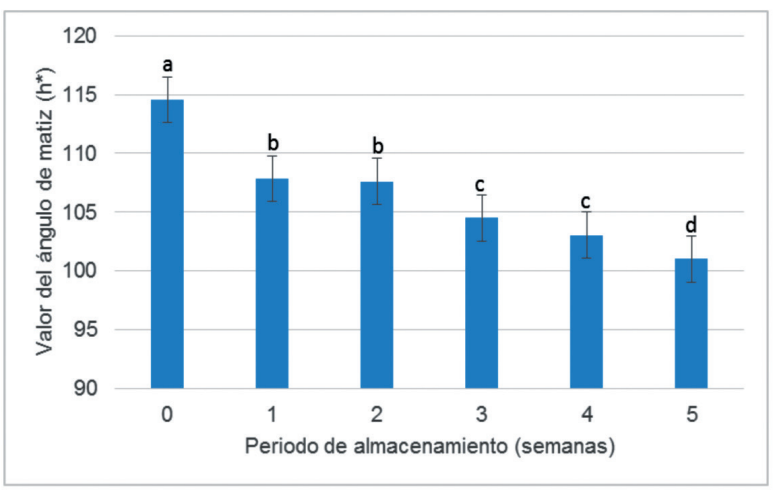

Figura 7. Efecto del periodo de almacenamiento a temperatura ambiente $\left(25 \pm 3{ }^{\circ} \mathrm{C}\right)$, sobre el ángulo de matiz $\left(\mathrm{h}^{*}\right)$ en tomate de cáscara, durante los ciclos verano-otoño 2013 y primavera-verano 2014. Oaxaca, México.

Letras distintas señalan diferencias significativas entre los valores según el método de Tukey $(\mathrm{p}<0,05)$.

Figure 7. Storage period at room temperature $\left(25 \pm 3{ }^{\circ} \mathrm{C}\right)$ effect on the hue angle $(\mathrm{h} *)$ in husk tomato varieties, in summer-autumn 2013 and springsummer 2014 cycles. Oaxaca, México.

Values with different letters are significantly different $(\mathrm{p}<0,05)$, by Tukey method.

las correspondientes a la semana 5 con 101, indicando que el tiempo de almacenamiento del fruto resultó en un cambio gradual de color de verde a amarillo (Figura 1). Resultados similares fueron obtenidos durante el proceso de maduración de jitomate, en el cual, el ángulo de tono presentó una tendencia a declinar, particularmente entre las etapas de maduración I y II (Radzevičius et al., 2008). En este mismo fruto, LópezCamelo y Gómez (2004), reportaron valores de h* de 113,3 para frutos verde-maduros (color verde) y de 59,3 para frutos en madurez de consumo (color rojo). De forma similar, Santamaría-Basulto et al. (2009b), reportaron un decremento en el valor de $\mathrm{h}^{*}$ con valores de 120 en el momento de corte, a 65 cuando el fruto alcanzó la madurez de consumo.

\section{Índice de color (IC)}

El análisis estadístico de los datos obtenidos para el índice de color, mostró diferencias significativas entre variedades (Figura 8), el valor más bajo ($6,0)$ fue para la variedad Diamante, seguida por las variedades San Martín y Rendidora con valores de $-5,75$ y finalmente Tecozautla 04 con $-5,67$. Esta tendencia fue similar a la descrita para el parámetro $\mathrm{a}^{*}$; sin embargo, en esta se formaron tres grupos diferentes de medias y en el caso de a* sólo se formaron dos. En general, los datos anteriores indican que los colores de los frutos estudiados fueron los característicos de estas variedades y van del verde profundo al verde amarillento, según la escala del IC (Vignoni et al., 2006).

La expresión de color fue modificada por el tiempo de almacenamiento de los frutos, el análisis estadístico definió seis grupos de medias (Figura 9), en el momento de corte los frutos alcanzaron un valor - 8,80, que aumentó moderadamente en la primera y segunda semana para obtener valores de $-6,94$ y $-5,67$, respectivamente; asimismo se incrementó de forma paulatina entre las semanas tres y cinco para alcanzar el valor final de -3,97. Lo que indica que el color de estos frutos cambió de verde profundo a verde amarillento como resultado de la degradación de la clorofila y síntesis de compuestos carotenoides (López-Camelo y Gómez, 2004; Vignoni et al., 2006).

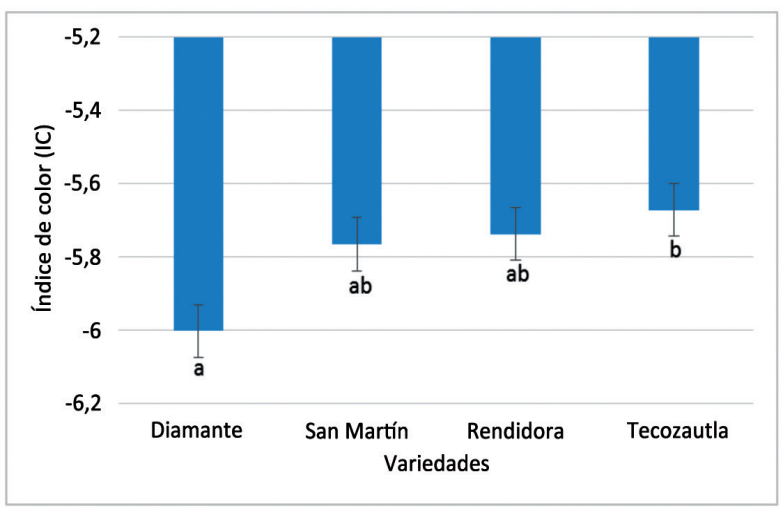

Figura 8. Valor del índice de color (IC) en variedades de tomate de cáscara almacenado a temperatura ambiente $\left(25 \pm 3{ }^{\circ} \mathrm{C}\right)$, después de la cosecha del fruto, durante los ciclos verano-otoño 2013 y primavera-verano 2014. Oaxaca, México. Letras distintas señalan diferencias significativas entre los valores según el Método de Tukey $(\mathrm{p}<0,05)$.

Figure 8. Colour index (CI) value in husk tomato varieties stored at room temperature $\left(25 \pm 3{ }^{\circ} \mathrm{C}\right)$, after the harvest of the fruit, in summer-autumn 2013 and spring-summer 2014 cycles. Oaxaca, México. Values with different letters are significantly different $(\mathrm{p}<0,05)$, by Tukey method. 


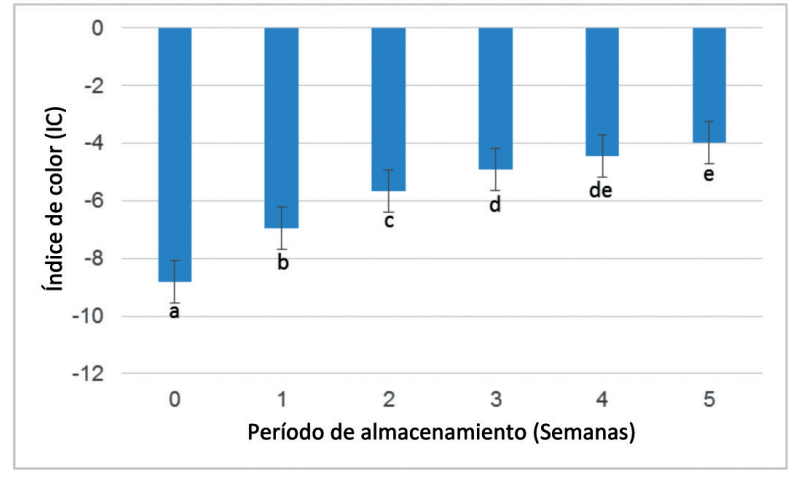

Figura 9. Efecto del periodo de almacenamiento a $25 \pm$ $3{ }^{\circ} \mathrm{C}$, sobre el valor del índice de color (IC) en variedades de tomate de cáscara, después de la cosecha del fruto, durante los ciclos verano-otoño 2013 y primavera-verano 2014. Oaxaca, México. Letras distintas señalan diferencias significativas entre los valores según el método de Tukey $(\mathrm{p}<0,05)$.

Figure 9. Storage period at $25 \pm 3^{\circ} \mathrm{C}$ effect on the colour index (CI) value in husk tomato varieties, after the harvest of the fruit, in summer-autumn 2013 and spring-summer 2014 cycles. Oaxaca, Mexico. Values with different letters are significantly different $(\mathrm{p}<0.05)$, by Tukey method.

Resultados similares de índice de color para jitomate (Lycopersicon esculentum Mill) fueron divulgados por López-Camelo y Gómez (2004), quienes encontraron una correlación directa entre esta expresión y el grado de maduración, con valores de IC de -14,6 para frutos verde maduros y de 21,8 para frutos en madurez de consumo.

\section{Acidez titulable}

Para las variedades de tomate estudiadas, el análisis estadístico de los datos de acidez de los frutos de tomate, marcó diferencias significativas entre las variedades (Figura 10), siendo la más alta la San Martín con 1,4\% de ácido cítrico, seguida por las otras tres con un promedio de 1,25\%. Esta variación en resultados pudo deberse a la variabilidad en el comportamiento fisiológico del género Physalis (Cantwell et al., 1992), por la manipulación genética a la que ha sido sometida en los últimos años (Peiris et al., 1997).

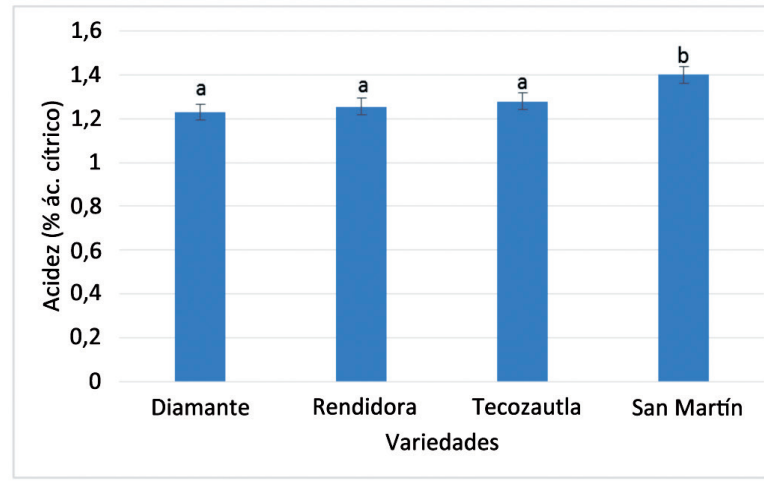

Figura 10. Acidez titulable en variedades de tomate de cáscara almacenado a temperatura ambiente ( 25 $\pm 3{ }^{\circ} \mathrm{C}$ ), después de la cosecha del fruto, durante los ciclos verano-otoño 2013 y primavera-verano 2014. Oaxaca, México.

Letras distintas señalan diferencias significativas entre los valores según el método de Tukey $(\mathrm{p}<0,05)$.

Figure 10. Titratable acidity in husk tomato varieties stored at room temperature $\left(25 \pm 3{ }^{\circ} \mathrm{C}\right)$, after the harvest of the fruit, in summer-autumn 2013 and springsummer 2014 cycles. Oaxaca, Mexico.

Values with different letters are significantly different $(\mathrm{p}<0.05)$, by Tukey method.

El contenido de acidez de los frutos fue modificado por el periodo de almacenamiento, y de acuerdo con el análisis de varianza, se encontró que este parámetro varió ligeramente del momento de corte hasta el final de las primeras dos semanas, alcanzando un promedio de 1,37\% de ácido cítrico. En este periodo su caída no fue significativa, pero posteriormente los valores descendieron hasta 1,26, 1,20 y 1,16\% de ácido cítrico (Figura 11); este cambio puede ser explicado por la síntesis de ácidos orgánicos en la primera semana y el bajo consumo de ácidos, debido a una tasa respiratoria también baja para este fruto (Peiris et al., 1997).

Este comportamiento es análogo al reportado para frutos de Physalis mínima L., en el cual, la acidez titulable presentó valores de $0,46,0,51$ y $0,47 \%$ de ácido cítrico en sus etapas de verde, premaduro y maduro, respectivamente (Patel et al., 2011). En frutos de Physalis peruviana L., Fisher y Martínez (1999), reportaron caídas de acidez de 40 a 15 meq de ácido cítrico/100g de tejido fresco para los estados de madurez verde y naranja-rojo, mutuamente. 


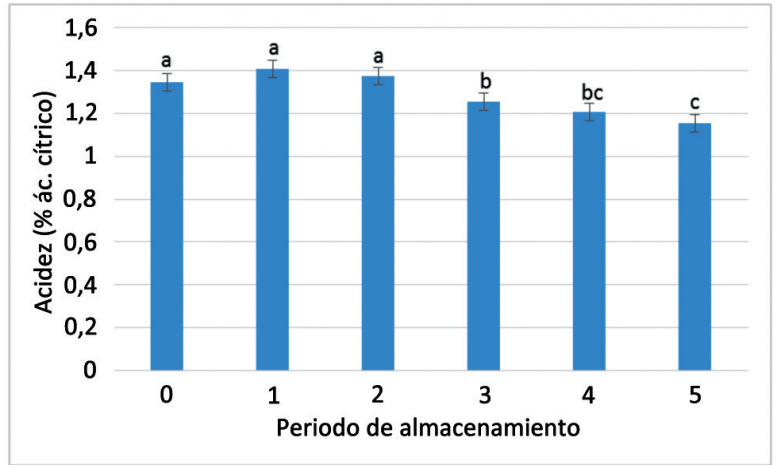

Figura 11. Efecto del periodo de almacenamiento a temperatura ambiente $\left(25 \pm 3{ }^{\circ} \mathrm{C}\right)$, sobre la acidez en variedades de tomate de cáscara, durante los ciclos verano-otoño 2013 y primavera-verano 2014. Oaxaca, México.

Letras distintas señalan diferencias significativas entre los valores según el método de Tukey $(\mathrm{p}<0,05)$.

Figure 11. Storage period at room temperature $\left(25 \pm 3{ }^{\circ} \mathrm{C}\right)$ effect on the acidity in husk tomato varieties, in summer-autumn 2013 and spring-summer 2014 cycles. Oaxaca, Mexico.

Values with different letters are significantly different $(\mathrm{p}<0,05)$, by Tukey method.

Resultados similares fueron encontrados en mango por Quintero et al. (2013), quienes reportaron un contenido de $1,3 \%$ de ácido cítrico en el momento de corte y de $0,3 \%$ después de nueve días de maduración a $25^{\circ} \mathrm{C}$.

\section{Pérdida de peso}

En relación a la pérdida de peso de los frutos, el análisis estadístico mostró diferencias significativas entre las variedades (Figura 12), correspondiendo a la variedad San Martín la menor pérdida con un promedio de $5,4 \%$, seguida de las variedades Tecozautla 04, Rendidora y Diamante con valores de $5,6,5,7$ y $5,9 \%$, respectivamente. Estos valores fueron modificados por el tiempo de almacenamiento. El análisis estadístico hizo una separación de medias de forma significativa para cada uno de las periodos de almacenamiento, obteniendo valores de $0,1,9$, $4,1,6,6,9,3$ y $12,0 \%$, desde el momento de corte hasta la quinta semana de almacén, respectivamente (Figura 13), marcando una tendencia lineal de esta variable en función al tiempo de almacenamiento. Lo anterior pudo deberse a la transpiración del fruto y su

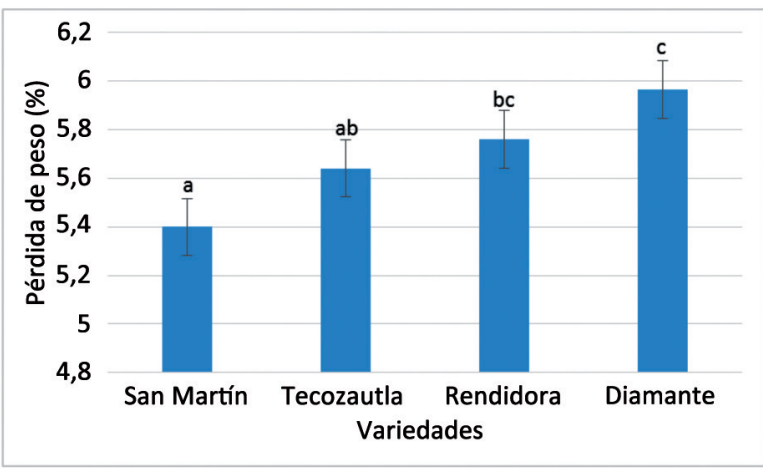

Figura 12. Pérdida de peso en variedades de tomate de cáscara almacenado a temperatura ambiente (25 $\pm 3{ }^{\circ} \mathrm{C}$ ), después de la cosecha del fruto, durante los ciclos verano-otoño 2013 y primavera-verano 2014. Oaxaca, México.

Letras distintas señalan diferencias significativas entre los valores según el método de Tukey $(\mathrm{p}<0,05)$.

Figure 12. Weight loss of husk tomato varieties stored at room temperature $\left(25 \pm 3{ }^{\circ} \mathrm{C}\right)$, after the harvest of the fruit, in summer-autumn 2013 and springsummer 2014 cycles. Oaxaca, Mexico.

Values with different letters are significantly different $(\mathrm{p}<0,05)$, by Tukey method.

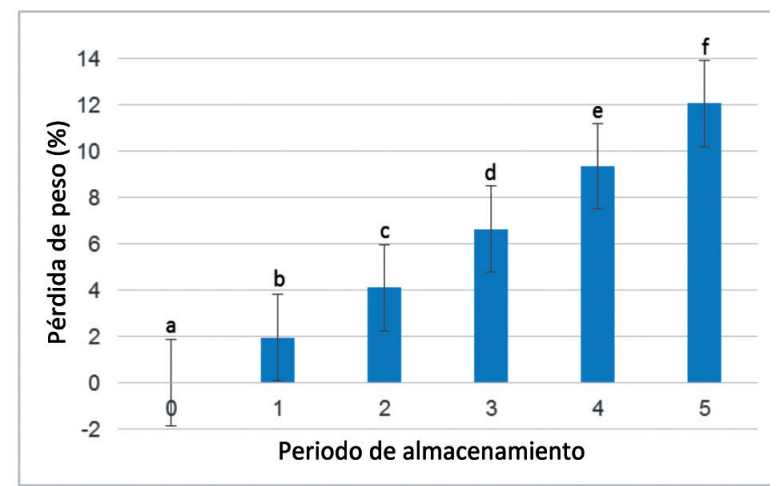

Figura 13. Efecto del periodo de almacenamiento $(25 \pm 3$ ${ }^{\circ} \mathrm{C}$ ), sobre la pérdida de peso en variedades de tomate de cáscara después de la cosecha del fruto., durante los ciclos verano-otoño 2013 y primaveraverano 2014. Oaxaca, México.

Letras distintas señalan diferencias significativas entre los valores según el método de Tukey $(\mathrm{p}<0,05)$.

Figure 13. Storage period $\left(25 \pm 3{ }^{\circ} \mathrm{C}\right)$ effect on the loss of weight of tomato husk varieties after the harvest of the fruit, in summer-autumn 2013 and springsummer 2014 cycles. Oaxaca, Mexico.

Values with different letters are significantly different $(\mathrm{p}<0,05)$, by Tukey method. 
consecuente pérdida de agua, al serle retirado el cáliz durante el periodo de almacenaje (Cruz-Álvarez et al., 2012). Resultados similares fueron descritos por Cruz-Álvarez et al. (2012) para la variedad "CFH1Chapingo", la cual alcanzó pérdidas de peso del 5,0\% después de ser almacenada por veinticinco días a 20 ${ }^{\circ} \mathrm{C}$. En la variedad "Tamazula" se reportaron pérdidas significativas de peso en frutos almacenados a 25 ${ }^{\circ} \mathrm{C}$, pero no para los conservados a $10{ }^{\circ} \mathrm{C}$ (GarcíaSahagún et al., 2007). De la misma forma, Cantwell et al. (1992) informaron pérdidas de peso en frutos de la variedad "Rendidora" cuando se almacenaron a 10 y $20^{\circ} \mathrm{C}$, comparadas con las de 5,0 y $2,5^{\circ} \mathrm{C}$, los cuales presentaron escasa pérdida de peso. Resultados similares fueron descritos para papaya, con pérdidas de peso de $13 \%$ después de seis días de maduración (Almeida-Castro et al., 2011).

\section{Sólidos solubles}

En este estudio, la cantidad de sólidos solubles $\left({ }^{\circ}\right.$ Brix) registró diferencias significativas entre las variedades (Figura 14), obteniéndose el valor más

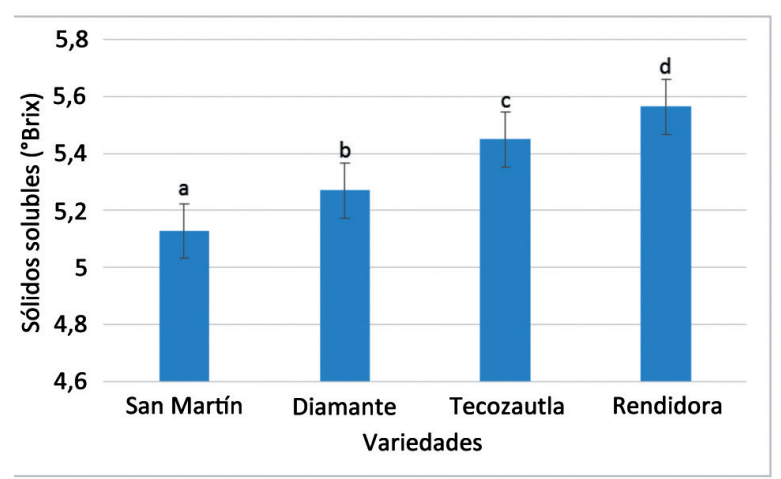

Figura 14. Contenido de sólidos solubles en variedades de tomate de cáscara almacenadas a temperatura ambiente $\left(25 \pm 3{ }^{\circ} \mathrm{C}\right)$, después de la cosecha del fruto, durante los ciclos verano-otoño 2013 y primavera-verano 2014. Oaxaca, México.

Letras distintas señalan diferencias significativas entre los valores según el método de Tukey $(\mathrm{p}<0,05)$.

Figure 14. Soluble solids content in husk tomato varieties stored at room temperature $\left(25 \pm 3{ }^{\circ} \mathrm{C}\right)$, after the harvest of the fruit, in summer-autumn 2013 and spring-summer 2014 cycles. Oaxaca, Mexico. Values with different letters are significantly different $(\mathrm{p}<0,05)$, by Tukey method. bajo para la variedad San Martín con 5,1, seguida por las variedades Diamante, Tecozautla 04 y Rendidora con valores de 5,27, 5,4 y 5,5, respectivamente. Los datos reportados son afines a los encontrados en cinco poblaciones autotetraploides y cuatro diploides por Ramírez-Godina et al. (2013), quienes indicaron un rango de 5,95 a $6,63{ }^{\circ}$ Brix, con diferencias significativas entre las poblaciones.

La cantidad de sólidos solubles en el tomate de cáscara fue modificada por el tiempo de almacenamiento, de acuerdo con la comparación de medias, los valores siguieron una pérdida paulatina desde el momento de corte hasta la quinta semana de almacén, con valores de 5,9 a 4,8 ${ }^{\circ}$ Brix (Figura 15). Este comportamiento es justificado por la escasa oxidación de azúcares y ácidos consumidos, debido a la baja tasa respiratoria de este fruto (Peiris et al., 1997). Los resultados fueron congruentes con la variabilidad natural de la especie y cercanos a los reportados para la variedad "CHF1- Chapingo", la cual tiene valores entre 6,1 y 5,25 que corresponden al momento de corte y después de veinticinco días de almacenamiento a $20^{\circ} \mathrm{C}$ (Cruz-Álvarez et al., 2012).

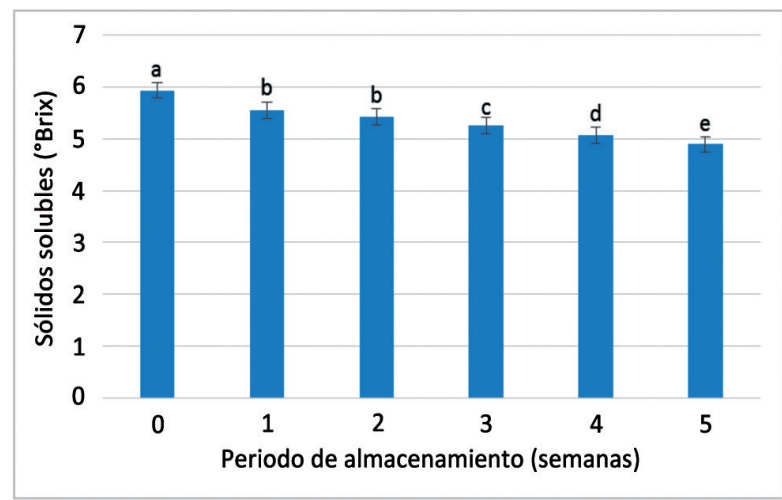

Figura 15. Efecto del periodo de almacenamiento a temperatura ambiente $\left(25 \pm 3{ }^{\circ} \mathrm{C}\right)$, sobre la cantidad de sólidos solubles en tomate de cáscara, después de la cosecha del fruto, durante los ciclos verano-otoño 2013 y primavera-verano 2014. Oaxaca, México. Letras distintas señalan diferencias significativas entre los valores según el método de Tukey $(\mathrm{p}<0,05)$.

Figure 15. Storage period effect at room temperature $(25 \pm$ $3{ }^{\circ} \mathrm{C}$ ), on the amount of soluble solids of tomato husk varieties after the harvest of the fruit, in summer-autumn 2013 and spring-summer 2014 cycles. Oaxaca, Mexico.

Values with different letters are significantly different $(\mathrm{p}<0,05)$, by Tukey method. 


\section{pH}

En este estudio se encontraron diferencias significativas $(\mathrm{p} \leq 0,05)$ para el $\mathrm{pH}$ entre las variedades, diferenciando a Diamante como la más ácida, con $\mathrm{pH}$ de 4,08, seguida por la San Martín con 4,10 y finalmente las variedades Rendidora y Tecozautla 04, formando el mismo grupo con valor de 4,13. Los datos de $\mathrm{pH}$ reportados se encuentran dentro del rango $(3,93$ - 4,23) obtenido para cinco poblaciones autotetraploides y cuatro diploides por Ramírez-Godina et al. (2013), cuyo análisis estadístico presentó diferencias significativas entre las poblaciones (Figura 16).

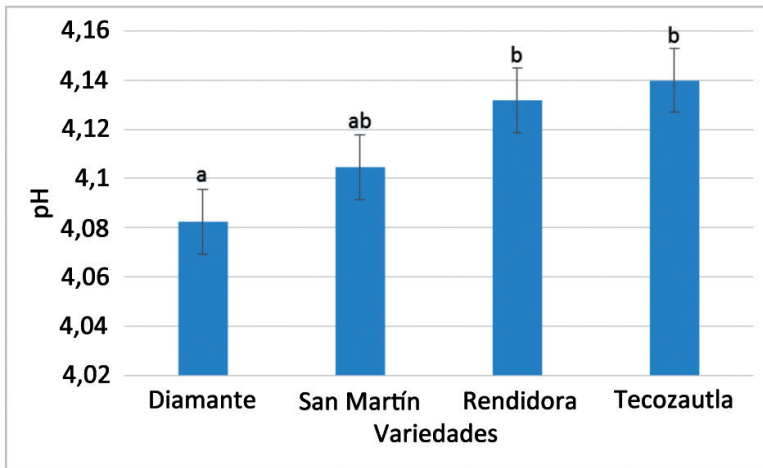

Figura 16. Valor de $\mathrm{pH}$ para variedades de tomate de cáscara almacenado a temperatura ambiente $\left(25 \pm 3^{\circ} \mathrm{C}\right)$, después de la cosecha del fruto, durante los ciclos verano-otoño 2013 y primavera-verano 2014. Oaxaca, México.

Letras distintas señalan diferencias significativas entre los valores según el método de Tukey $(\mathrm{p}<0,05)$.

Figure 16. $\mathrm{pH}$ value of husk tomato varieties stored at room temperature $\left(25 \pm 3^{\circ} \mathrm{C}\right)$, after the harvest of the fruit, in summer-autumn 2013 and spring-summer 2014 cycles. Oaxaca, Mexico.

Values with different letters are significantly different $(\mathrm{p}<0,05)$, by Tukey method.

Al igual que otras características, el $\mathrm{pH}$ fue modificado durante el periodo de almacenamiento (Figura 17). El análisis estadístico mostró diferencias significativas entre las diferentes fechas de almacén, el valor inicial en el momento de corte fue de 3,60, el cual se incrementó gradualmente para alcanzar el valor de 4,46 en la quinta semana de almacenamiento.

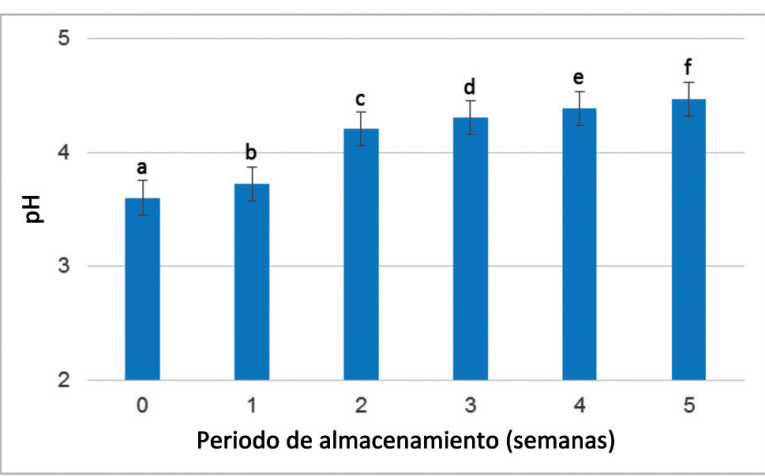

Figura 17. Efecto del periodo de almacenamiento sobre el $\mathrm{pH}$ en variedades en tomate de cáscara, almacenadas a temperatura ambiente $\left(25 \pm 3{ }^{\circ} \mathrm{C}\right)$, durante los ciclos verano-otoño 2013 y primavera-verano 2014. Oaxaca, México.

Letras distintas señalan diferencias significativas entre los valores según el método de Tukey $(\mathrm{p}<0,05)$.

Figure 17. Storage period effect on the $\mathrm{pH}$ in husk tomato varieties, stored at room temperature $\left(25 \pm 3{ }^{\circ} \mathrm{C}\right)$, in summer-autumn 2013 and spring-summer 2014 cycles. Oaxaca, Mexico.

Values with different letters are significantly different $(\mathrm{p}<0,05)$, by Tukey method.

Este comportamiento puede explicarse por la síntesis y acumulación de ácidos orgánicos hasta el momento de corte y su pérdida debida a la respiración, durante la maduración.

Los datos encontrados fueron similares a los reportados por Jiménez-Santana et al. (2012), quienes señalaron un rango de 3,51 a 4,51, entre tres genotipos tetraploides y la variedad diploide "Rendidora" con diferencias significativas por efecto de la fecha de corte en los tetraploides. Esta misma tendencia ha sido encontrada en jitomate (Juárez-López et al., 2009), en Physalis peruviana L. (Lanchero et al., 2007) y varios frutos tropicales, a excepción del plátano (Torres et al., 2013).

\section{Resistencia a la penetración}

El análisis estadístico aplicado a los valores encontrados de resistencia a la penetración para las variedades estudiadas, mostraron diferencias significativas $(\mathrm{p} \leq 0,05)$ entre variedades. La variedad Rendidora se presentó con la menor resistencia, con un valor de 33,9 N, seguida por Diamante con 37,0 N; 
mientras que San Martín y Tecozautla 04 fueron las más resistentes al manejo poscosecha con valores similares de 39,0 N (Figura 18).

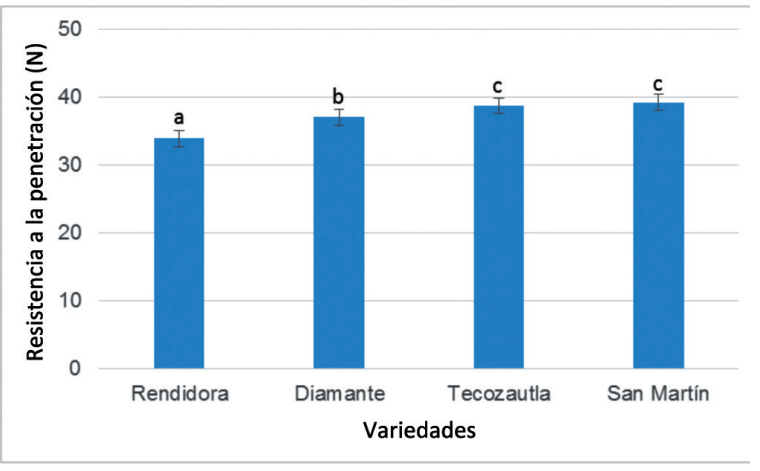

Figura 18. Resistencia a la penetración en variedades de tomate de cáscara almacenadas a temperatura ambiente $\left(25 \pm 3{ }^{\circ} \mathrm{C}\right)$, durante los ciclos veranootoño 2013 y primavera-verano 2014. Oaxaca, México.

Letras distintas señalan diferencias significativas entre los valores según el método de Tukey $(\mathrm{p}<0,05)$.

Figure 18. Penetration resistance in husk tomato varieties stored at room temperature $\left(25 \pm 3{ }^{\circ} \mathrm{C}\right)$, in summer-autumn 2013 and spring-summer 2014 cycles. Oaxaca, Mexico.

Values with different letters are significantly different $(\mathrm{p}<0,05)$, by Tukey method.

La información obtenida del análisis estadístico mostró que hay diferencias significativas entre las etapas del periodo de almacenamiento evaluadas, el valor inicial de la resistencia en el momento de corte fue de $46,3 \mathrm{~N}$, el cual decayó hasta alcanzar 27,2 $\mathrm{N}$ en la quinta semana de almacenaje (Figura 19), estos resultados se debieron a la solubilización de las sustancias pécticas que forman parte estructural de la pared celular, por efecto del incremento de la actividad de enzimas como la poligalacturonasa y la celulasa (Barret et al., 1998).

Resultados similares de resistencia a la penetración fueron reportados por García-Sahagún et al. (2007) para otras variedades de tomate de cáscara, quienes mencionan valores de 44,1 $\mathrm{N}$ para frutos en el momento de corte, y de 14,7 $\mathrm{N}$ después de dos semanas de almacenamiento a $25^{\circ} \mathrm{C}$. En uchuva (Physalis peruviana L.) se han reportado valores equivalentes a $7,5 \mathrm{~N}$ al momento de corte y de 5,8 $\mathrm{N}$ después de cuatro días de

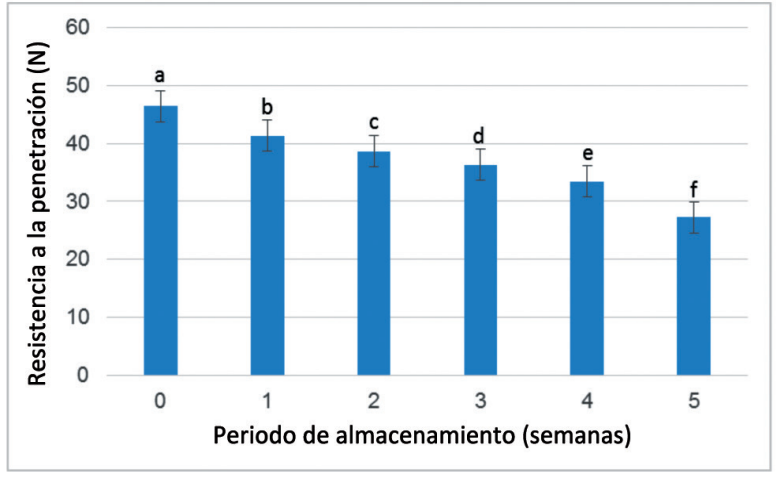

Figura 19. Efecto del periodo de almacenamiento a $(25 \pm$ $3{ }^{\circ} \mathrm{C}$ ), sobre la resistencia a la penetración en variedades de tomate de cáscara, durante los ciclos verano-otoño 2013 y primavera-verano 2014. Oaxaca, México.

Letras distintas señalan diferencias significativas entre los valores según el método de Tukey $(\mathrm{p}<0,05)$.

Figure 19. Storage period at $\left(25 \pm 3{ }^{\circ} \mathrm{C}\right)$ effect on the penetration resistance in husk tomato varieties, in summer-autumn 2013 and spring-summer 2014 cycles. Oaxaca, Mexico.

Values with different letters are significantly different $(\mathrm{p}<0,05)$, by Tukey method.

almacenamiento (Lanchero et al., 2007), lo cual significa que este fruto tiene una resistencia a la penetración menor al tomate de cáscara. En tomate se han reportado pérdidas de firmeza que van de $11,65 \mathrm{~N}$ en el momento de corte, a 4,0 $\mathrm{N}$ en puntos de comercialización (Zapata et al., 2007; Oms-Oliub et al., 2011).

\section{Relación entre variables}

Los coeficientes de correlación entre los diferentes parámetros de color analizados, mostraron similitudes estadísticamente significativas en tres de ellos: $a^{*}, h^{*}$ e IC (Cuadro 1), el valor de $C^{*}$ no presentó analogía con el resto de las expresiones de color. A su vez, de los tres parámetros de color, el parámetro a* mostró el mayor coeficiente de correlación con la resistencia a la penetración del fruto, característica que más se asocia a la calidad del fruto, seguida por el IC y después el valor $\mathrm{h}^{*}$. En el caso de la pérdida de peso, los coeficientes de correlación fueron muy similares para $\mathrm{a}^{*}$ e IC y un poco menor con $\mathrm{h}^{*}$. Esta relación se repitió para las variables acidez, sólidos solubles y pH.

La calidad de los frutos fue afectada por los factores genéticos, por el manejo recibido durante 
Cuadro 1. Coeficientes de correlación entre los parámetros de color, y características físicas y químicas en variedades de tomate de cáscara durante su almacenamiento a temperatura ambiente $\left(25 \pm 3{ }^{\circ} \mathrm{C}\right)$, durante los ciclos verano-otoño 2013 y primavera-verano. Oaxaca, México. 2014.

Table 1. Correlation coefficients among parameters of color, and physical and chemical properties in husk tomato varieties during storage at room temperature $\left(25 \pm 3{ }^{\circ} \mathrm{C}\right)$, in summer-autumn 2013 and spring-summer 2014 cycles. Oaxaca, Mexico.

\begin{tabular}{lccccccccc}
\hline & Acidez & $\mathbf{P P}$ & ${ }^{\circ}$ Brix & $\mathbf{p H}$ & Textura & $\mathbf{a}^{*}$ & $\mathbf{C}^{*}$ & $\mathbf{h}^{*}$ & $\mathbf{I C}$ \\
\hline Acidez & & $-0,504$ & 0,311 & $-0,484$ & 0,469 & $-0,433$ & $-0,231$ & 0,437 & $-0,385$ \\
Valor P & & 0,000 & 0,000 & 0,000 & 0,000 & 0,000 & 0,000 & 0,000 & 0,000 \\
$\mathrm{PP}$ & $-0,504$ & & $-0,753$ & 0,938 & $-0,833$ & 0,831 & 0,243 & $-0,792$ & 0,835 \\
Valor P & 0,000 & & 0,000 & 0,000 & 0,000 & 0,000 & 0,000 & 0,000 & 0,000 \\
${ }^{\circ}$ Brix & 0,311 & $-0,753$ & & $-0,717$ & 0,543 & $-0,579$ & $-0,164$ & 0,604 & $-0,647$ \\
Valor P & 0,000 & 0,000 & & 0,000 & 0,000 & 0,000 & 0,011 & 0,000 & 0,000 \\
pH & $-0,484$ & 0,938 & $-0,717$ & & $-0,782$ & 0,767 & 0,278 & $-0,729$ & 0,802 \\
Valor P & 0,000 & 0,000 & 0,000 & & 0,000 & 0,000 & 0,000 & 0,000 & 0,000 \\
Textura & 0,469 & $-0,833$ & 0,543 & $-0,782$ & & $-0,784$ & $-0,137$ & 0,753 & $-0,723$ \\
Valor P & 0,000 & 0,000 & 0,000 & 0,000 & & 0,000 & 0,034 & 0,000 & 0,000 \\
a* & $-0,433$ & 0,831 & $-0,579$ & 0,767 & $-0,784$ & & 0,039 & $-0,802$ & 0,801 \\
Valor P & 0,000 & 0,000 & 0,000 & 0,000 & 0,000 & & 0,542 & 0,000 & 0,000 \\
C* & $-0,231$ & 0,243 & $-0,164$ & 0,278 & $-0,137$ & 0,039 & & $-0,258$ & 0,376 \\
Valor P & 0,000 & 0,000 & 0,011 & 0,000 & 0,034 & 0,542 & & 0,000 & 0,000 \\
h* & 0,437 & $-0,792$ & 0,604 & $-0,729$ & 0,753 & $-0,802$ & $-0,258$ & & $-0,817$ \\
Valor P & 0,000 & 0,000 & 0,000 & 0,000 & 0,000 & 0,000 & 0,000 & & 0,000 \\
IC & $-0,385$ & 0,835 & $-0,647$ & 0,802 & $-0,723$ & 0,801 & 0,376 & $-0,817$ & \\
Valor P & 0,000 & 0,000 & 0,000 & 0,000 & 0,000 & 0,000 & 0,000 & 0,000 & \\
\hline
\end{tabular}

el cultivo, cosecha y poscosecha, y por el grado de madurez en el momento de consumo (Kader, 2001). El sabor de los frutos depende del gusto, un balance entre dulzor, acidez, astringencia y aroma; muchos de estos factores están relacionados con el color del epicarpio del mismo (López-Camelo y Gómez, 2004). En el caso de las variedades de tomate de cáscara las expresiones más significativas se encontraron con la coordenada $\mathrm{a}^{*}, \mathrm{~h}^{*} \mathrm{e}$ IC, que indicaron esencialmente lo mismo; sin embargo, tienen diferencia en cuanto a la percepción humana. Croma no se manifestó como un buen parámetro para expresar la madurez de tomate de cáscara. Las expresiones de color a*, $\mathrm{h}^{*}$ e IC, presentaron alta correlación con dos factores que determinan su calidad, a saber, la pérdida de textura y de peso, y otros factores que intervienen en el gusto del consumidor como la acidez y los sólidos solubles. Por la facilidad para detectar el color verde en el fruto, el parámetro a* fue el que presentó mayor probabilidad de ser utilizado para medir la calidad del fruto. De acuerdo con los resultados obtenidos, las variedades con mejores características de calidad fueron Tecozautla 04 y San Martín.

\section{LITERATURA CITADA}

Almeida-Castro, A., J.D. Reis-Pimentel, D. Santos-Souza, T. Vieira de Oliveira, y M. da Costa-Oliveir. 2011. Estudio de la conservación de la papaya (Carica papaya L.) asociado a la aplicación de películas comestibles. RVCTA 2:49-60. 
Amnuaysin, N., K. Seraypheap, and M. Kidyoo. 2012. Anatomical changes in peel structure of 'Hom Thong' banana during fruit development and ripening. Trop. Nat. Hist. 12:127-136.

AOAC (Association of Official Analytical Chemists). 2010. Official Methods of AOAC. 18a ed. AOAC International, Gaithersburg, MD, USA.

Ayala-Silva,T., R.J.Schnel, A.W.Meerow, M. Winterstein, C. Cervantes, and J. Steven Brown. 2005. Determination of color and fruit traits of half-sib families of mango (Mangifera indica L.). Proc. Fla State Hortic. Soc. 118:253-257.

Azcón Bieto, J., y M. Talón. 2000. Fundamentos de fisiología vegetal. Ediciones Universitarias de Barcelona, ESP.

Bao-Ning, S., R. Misico, E. Jung-Park, B.D. Santarsiero, A.D. Mesecar, H.H.S. Fong, J.M. Pezzuto, and A.D. Kinghorn. 2002. Isolation and characterization of bioactive principles of the leaves and stems of Physalis philadelphica. Tetrahedron 58:3453-3466.

Barrett, D.M., E. García, and J.E. Wayne. 1998. Textural modification of processing tomatoes. Crit. Rev. Food Sci. Nutr. 38:173- 258.

Cantwell, M., J. Flores-Minutti, and A. Trejo-González. 1992. Developmental changes and postharvest physiology of tomatillo fruit (Physalis ixocarpa Brot). Sci. Hortic. 50:59-70.

Carvajal-Herrera, J.J, I.D. Aristizábal-Torres, C.E. OliverosTascón, y J.W. Mejía-Montoya. 2011 . Colorimetría del fruto de café (Coffea arabica L.) durante su desarrollo y maduración. Rev. Fac. Nal Agr. Medellín 64:62296240

Choi, J.K., G. Murillo, J.S. Bao-Ning, J.M. Pezzuto, A.D. Kinghorn, and R.G. Mehta. 2006. Ixocarpalactone A isolated from the Mexican tomatillo shows potent antiproliferative and apoptotic activity in colon cancer cells. FEBS J. 273:5714-5723.

Cruz-Álvarez, O., M.T. Martínez-Damián, J.E. RodríguezPérez, M.T. Colinas-León, y E. del C. Moreno-Pérez. 2012. Conservación poscosecha de tomate de cáscara (Physalis ixocarpa Brot. ex Horm.) con y sin cáliz. Rev. Chapingo Ser. Hortic. 18:333-344.

Dávila-Aviña, J.E. de J., G.A. González-Aguilar, J.F Ayala-Zavala, D.R. Sepúlveda, y G.I. Olivas. 2011. Compuestos volátiles responsables del sabor del tomate. Rev. Fitotec. Mex. 34:133-143.

Fischer G., y O. Martínez, 1999. Calidad y madurez de uchuva (Physalis peruviana L.) en relación con la coloración del fruto. Agron. Colomb. 16(1-3):35-39.
Francis, F.J., and F.M. Clydesdale. 1975. Food colorimetry: Theory and applications. The AVI Pub. Co., CT, USA.

García-Sahagún, M.L., A.N. Avendaño-López, J.N. Mercado-Ruiz, y L. Robles-Osuna. 2007. Calidad poscosecha de frutos de tomate de cáscara (Physalis spp.) durante almacenamiento. En: S. Carvajal, y E. Pimienta Barrios, editores, Avances en la Investigación Científica en el CUCBA. XVIII Seminario de la Investigación Científica. Centro Universitario de Ciencias Biológicas y Agropecuarias. Universidad de Guadalajara, MEX.p. 1-5.

González-Cárdenas, I.A., C. Osorio, F J. Rodríguez-Pulido, M.L. González-Miret, y F.J. Heredia. 2010. Color y apariencia de frutos de guayaba a lo largo de la maduración. En: Universidad de Alicante, editor, Memorias del IX Congreso Nacional del Color, Alicante, 29 y 30 de junio, 1 y 2 de julio del 2010, Alicante, ESP. p. 316-319.

González-Mendoza, D., D. Ascencio-Martínez, A. Hau Poox, V. Méndez-Trujillo, O. Grimaldo-Juárez, J. F. Santiaguillo Hernández, L. Cervantes-Díaz, and S.M. Avilés-Marin. 2011. Phenolic compounds and physiochemical analysis of Physalis ixocarpa genotypes. Sci. Res. Essays 6:3808-3814.

Hornero-Méndez, D., and M.I. Mínguez-Mosquera. 2002. Chlorophyll disappearance and chlorophyllase activity during ripening of Capsicum annuum L fruits. J. Sci. Food Agric. 82:1564-1570.

Ibave-González, J.L., y M. Ochoa. 2007. Cuantificación de los diferentes folatos presentes en tomatillo (Physalis ixocarpa) por cromatografía de líquidos de alta resolución. TECNOCIENCIA Chihuahua 1:9-16.

Jha, S.N., A.R.P. Kingsly, and S. Chopra. 2006. Physical and mechanical properties of mango during growth and storage for determination of maturity. J. Food Eng. 72:73-76.

Jha, S.N., K. Narsaiah, A.D. Sharma, M. Singh, S. Bansal, and R. Kumar. 2010. Quality parameters of mango and potential of non-destructive techniques for their measurement: A review. J. Food Sci. Technol. 47:1-14.

Jiménez-Santana, E., V. Robledo-Torres, A. BenavidesMendoza, F. Ramírez-Godina, H Ramírez-Rodríguez, y E. de la Cruz-Lázaro. 2012. Calidad de fruto de genotipos tetraploides de tomate de cáscara (Physalis ixocarpa Brot.). Universidad y Ciencia 28(2):153-161.

Juárez-López P., R. Castro-Brindis, T. Colinas-León, P. Ramírez-Vallejo, M. Sandoval-Villa, D.W. Reed, L. Cisneros-Zevallos, y S. King. 2009. Evaluación de 
calidad de frutos de siete genotipos nativos de jitomate (Lycopersicon esculentum var. cerasiforme). Rev. Chapingo Ser. Hortic. 15(esp):5-9.

Kader, A.A. 2001. Quality assurance of harvested horticultural perishables. Acta Hort. 553:51-55.

Kader,A.A. 2009. La calidad del sabor de frutas y hortalizas. Hortic. Internacional 69:6-7.

Karakurt, Y. 2007. Fruit Cell Wall polysacharides and their modification during ripening. J. Agric. Fac. HR. 11(1/2):13-19.

Lancaster, J.E., C.E. Lister, P.F. Reay, and C.M. Triggs. 1997. Influence of pigment composition on skin color in a wide range of fruit and vegetables. J. Amer. Soc. Hort. Sci. 122:594-598.

Lanchero, O., G. Velandia, G. Fischer, N.C. Varela, y H. García. 2007. Comportamiento de la uchuva (Physalis peruviana L.) en poscosecha bajo condiciones de atmósfera modificada activa. Rev. Corpoica 8(1):61-68.

León, K., D. Mery, F. Pedreschi, and J. León. 2006. Color measurement in $\mathrm{L} * \mathrm{a} * \mathrm{~b} *$ units from RGB digital images. Food Res. Int. 39:1084-1091.

López-Camelo, A.F., and P. A. Gómez. 2004. Comparison of color indexes for tomato ripening. Hortic. Bras. 22:534-537.

McGuirre, R.G. 1992. Reporting of objective color measurements. Hort. Sci. 27:1254-1255.

Oms-Oliub, G., M.L.A.T.M. Hertoga, B. Van de Poel, J. Ampofo-Asiamaa, A.H. Geeraerda, and B.M. Nicolaï. 2011. Metabolic characterization of tomato fruit during preharvest development, ripening, and postharvest shelf-life. Postharvest Biol. Technol. 62:7-16.

Osuna-García, J.A., V. Vázquez-Valdivia, y M.H. PérezBarraza. 2007. Caracterización postcosecha de cultivares de plátano para consumo en fresco. Rev. Chapingo Ser. Hortic. 14:139-145.

Padda, M.S., C.V.T. do Amaranteb, R.M. Garcia, D.C. Slaughterd, and E.J. Mitchama. 2011. Methods to analyze physico-chemical changes during mango ripening: A multivariate approach. Postharvest Biol. Technol. 62:267-274.

Patel, P.R., N.B. Gol, and T.V. Ramana-Rao. 2011. Physiochemical changes in sunberry (Physalis minima L.) fruit during growth and ripening. Fruit 66:37-46.

Pathare, P.B., U.L. Opara, and F.J. Al-Said. 2013. Colour Measurement and analysis in fresh and processed foods: a review. Food Bioprocess Technol. 6:36-60.

Peiris, K.H.S., J.L. Mallon, and S.J. Kays. 1997. Respiratory rate vital heat of some specialty vegetables at various storage temperatures. HorTechnology 7:46-49.
Pérez-López, A., C.A. Villaseñor-Pérez, V. CrisantoMartínez, y J.J.E. Corrales-García. 2009. Propiedades mecánicas y maduración de frutos de mango (Mangifera indica L.) bajo compresión axial. INAGBI 1:19-23.

Quintero. C.V., G. Giraldo, J. Lucas, y J. Vasco. 2013. Caracterización fisicoquímica del mango común (Mangifera indica L.) durante su proceso de maduración. Biotecnol. Sector Agropec. Agroind. 11(1):10-18.

Radzevičius, A., P. Viškelis, and Č. Bobinas. 2008. Quality and physiological parameters of tomato (Lycopersicon esculentum Mill.) fruits of Lithuanian selection. Biologija 54:108-111.

Ramírez-Godina, F., V. Robles-Torres, R. ForoughbakhchPournabav, A. Benavides-Mendoza, J.L. HérnándezPiñero, M.H. Reyes-Váldez, and M.A. AlvaradoVázquez. 2013. Yield and fruit quality evaluation in husk tomato autotetraploids (Physalis ixocarpa) and diploids. Aust. J. Crop Sci. 7:933-940.

Salvador, A., T. Sanz, and S.M. Fiszman. 2007. Changes in colour and texture and their relationship with eating quality during storage of two different dessert bananas. Postharvest Biol. Technol. 43:319-325.

Santamaría-Basulto, F., R. Díaz-Plaza, E. Sauri-Duch, F. Espadas y Gil, J.M. Santamaría-Fernández, y A. Larqué-Saavedra. 2009a. Características de calidad de frutos de papaya Maradol en la madurez de consumo. Agric. Téc. Méx. 35:347-353.

Santamaría-Basulto, F., E. Sauri-Duch, F. Espadas y Gil, R. Díaz-Plaza, A. Larqué-Saavedra, and J.M. Santamaría. 2009b. Postharvest ripening and maturity indices for Maradol papaya. Interciencia 34:583-588.

Santiaguillo-Hernández, J.F., y S. Blas-Yáñez. 2009. Aprovechamiento tradicional de las especies de Physalis en México. Rev. Geograf. Agríc. 43:81-86.

Santiaguillo-Hernández, J.F., T. Cervantes-Santana, y A. Peña-Lomelí. 2004. Selección para rendimiento $\mathrm{y}$ calidad de fruta de cuzas planta $\mathrm{x}$ planta entre variedades de tomate de cáscara. Rev. Fitotec. Mex. 27:84-91.

SECOFI (Secretaria de Comercio y Fomento Industrial). 1982. Norma Mexicana NMX-FF-54-1982. Productos alimenticios no industrializados para uso humano - hortalizas en estado fresco-tomate con cáscara. SECOFI, MEX.

Soltani M., R. Alimardani, and M. Omid. 2011. Changes in physico-mechanical properties of banana fruit during ripening treatment. J. Am. Sci. 7:14-19 
Surmacka, S.A. 2002. Texture is a sensory property. Food Qual. Prefer. 13:215-225.

Tapre, A.R., and R.K. Jain 2012. Study of advanced maturity stages of banana. Int. J. Adv. Eng. Res. Stud. I (III):272-274

Torres, R., E.J. Montes, O. Pérez, y R.D. Andrade. 2013. Relación del color y del estado de madurez con las propiedades fisicoquímicas de frutas tropicales. Inf. Tecnol. 24(3):51-56.

Torres-Panduro, V. 1998. Componentes de calidad en cuarenta materiales de tomate de cascara (Physalis ixocarpa Brot.) Tesis Ing. Agr., Universidad de Guadalajara, Las Agujas, Zapopan, Jalisco, MEX.
Vignoni, L.A., R.M. Cesari, M. Forte, y M.L. Mirábile 2006. Determinación de índice de color en ajo picado. Inf. Tec. 17(6):63-67.

Yam, K.L., and S. Papadakis. 2004. A simple digital imaging method for measuring and analyzing color of food surfaces. J. Food Eng. 61:137-142.

Zapata, L., L. Gerard, C. Davies, L. Oliva, y M. Schva. 2007. Correlación matemática de índices de color del tomate con parámetros texturales y concentración de carotenoides. Cienc. Docencia Tecnol. XVIII (34):207-226. 